\title{
Einfache und pragmatische Risikoabschätzung
}

Fragestellung: Wie kann das Risiko einer progressiven multifokalen Leukenzephalopathie (PML) unter der Therapie mit Natalizumab abgeschätzt und wie können die Patienten überwacht werden?

Hintergrund: Natalizumab ist eine hocheffektive Therapie für die aktive schubförmige Multiple Sklerose (MS). Eine seltene, aber gefürchtete Nebenwirkung der Therapie ist das Auftreten einer PML. Eine Früherkennung mittels kranieller Magnetresonanztomografie (cMRT) kann eventuell die Prognose der Erkrankung verbessern.

McGuigan C, Craner M, Guadagno J et al. Stratification and monitoring of natalizumabassociated progressive multifocal leukoencephalopathy risk: recommendations from an expert group. J Neurol Neurosurg Psychiatry 2016; 87: 117-25
Patienten und Methodik: Es handelt sich um einen in Großbritannien und Irland erarbeiteten Expertenkonsens für Neurologen, die Natalizumab in der Praxis anwenden. Die Empfehlungen basieren auf aktuellen Erkenntnissen zur Wertigkeit des Anti-JohnCunningham-Virus (JCV)-
Antikörperindex bei MS-Patienten ohne immunsuppressive Vortherapie, die mit Natalizumab behandelt werden oder behandelt werden sollen.

Ergebnisse: Ist der Anti-JCV-Antikörperstatus negativ oder der Index $\leq 1,5$, sollte der Test unter Therapie alle sechs Monate erneut durchgeführt werden. Bei einem Index $>1,5$ ist das nicht notwendig. Bei negativem Anti-JCV-Antikörperstatus werden cMRT-Untersuchungen einmal pro Jahr empfohlen. Ist der In$\operatorname{dex}<1,5$, so beträgt das PML-Risiko im dritten und vierten Therapiejahr 1:885. In dieser Situation sollen cMRT-Untersuchungen ab dem 18. Therapiemonat sechsmonatlich durchgeführt werden. $\mathrm{Ab}$ einem Index von $>1,5$ beträgt das PML-Risiko im dritten und vierten Jahr $1: 113$. Hier ist die cMRT-Frequenz ab dem 18. Therapiemonat auf vierteljährliche Abstände zu erhöhen.

Schlussfolgerungen: Die Übersicht liefert einen einfachen und pragmatischen Algorithmus zum Einsatz des Anti-JCV-Antikörperindex sowie eines cMRT-Monitoring als PML-Vorsorge. Dieser Standard kann zur individuellen Begleitung von MS-Patienten dienen, die trotz Anti-JCV-Seropositivität eine Therapie mit Natalizumab beginnen oder fortführen wollen.

\section{- Kommentar von Ralf Linker, Erlangen}

\section{Vigilanz bleibt wichtig}

Die Übersichtsarbeit fasst die dem aktuellen Wissensstand entsprechende Risikostratifizierung und PML-Vorsorge unter der Therapie mit Natalizumab in einem Flussdiagramm schön zusammen. Auch in Deutschland wird eine ähnliche Praxis bereits vielfach umgesetzt und in der TRUST-Studie mit einer Vorreiterrolle in Europa auch beobachtend untersucht. Die TRUST-Studie ist eine prospektive, nicht interventionelle, multizentrische, nationale Kohortenstudie, die an 200 Zentren 1.260 Patienten mit schubförmiger MS und wenigstens zwölfmonatiger Natalizumab-Therapie einschließt. Die Patienten werden dann über drei Jahre nachbeobachtet. Die Beobachtungsstudie läuft seit Frühjahr 2014 und wird die Rekrutierung

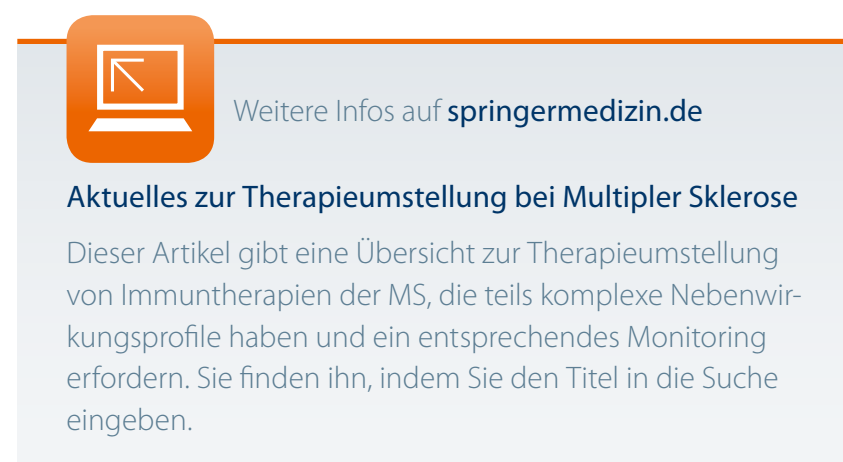

voraussichtlich Mitte 2016 abschließen. Einschränkend ist zu bemerken, dass das hier im Expertenkonsens vorgeschlagene Vorgehen nicht prospektiv validiert ist. Bis heute existiert keine eindeutige Evidenz, ob ein solcher Algorithmus auf individueller Ebene tatsächlich das Auftreten einer PML verhindert oder den Outcome im Falle einer PML verbessert. Eine interventionelle Studie hierzu ist aufgrund der niedrigen Ereignisfrequenz nicht sinnvoll umsetzbar. Eine umfassende klinische Vigilanz und regelmäßige Patientenaufklärung sind daher weiterhin zentrale Punkte in der Anwendung hochaktiver MSImmuntherapien. Es bleibt abzuwarten, wie sich derartige Algorithmen weiter anpassen werden, wenn zukünftig möglicherweise weitere monoklonale Antikörper wie Daclizumab oder auch Ocrelizumab für die Therapie der aktiven MS zur Verfügung stehen.

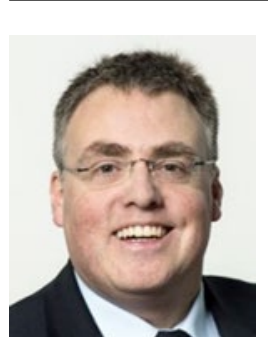

Prof. Dr. med. Ralf Linker, Erlangen

Neurologie, Neuromuskuläres Zentrum, Universitätsklinikum Erlangen E-Mail: ralf.linker@uk-erlangen.de 